\title{
The EVN view of the highly variable TeV active galaxy IC 310
}

\author{
Robert Schulz $^{* a, b}$, Matthias Kadler ${ }^{a}$, Eduardo $\operatorname{Ros}^{c, d}$, Dorit Eisenacher Glawion ${ }^{a}$, \\ Uwe Bach $^{c}$, Dominik Elsässer ${ }^{a}$, Christoph Grossberger ${ }^{e, b}$, Ingo Kreykenbohm ${ }^{b}$, \\ Karl Mannheim $^{a}$, Cornelia Müller ${ }^{a, b}$, Jonas Trüstedt ${ }^{a}$, Jörn Wilms ${ }^{b}$ \\ ${ }^{a}$ Lehrstuhl für Astronomie, Universität Würzburg, Campus Hubland Nord, Emil-Fischer-Strasse \\ 31, 97074 Würzburg, Germany \\ ${ }^{b}$ Dr. Remeis Sternwarte \& ECAP, Universität Erlangen-Nürnberg, Sternwartstr. 7, 96049 \\ Bamberg, Germany \\ ${ }^{c}$ Max-Planck-Institut für Radioastronomie, Auf dem Hügel 69, 53121 Bonn, Germany \\ ${ }^{d}$ Observatori Astronòmic \& Departament d'Astronomia i Astrofísica, Universitat de València, \\ 46071 València, Spain \\ ${ }^{e}$ Max-Planck-Institut für extraterrestrische Physik, Giessenbachstrasse 1, 85741 Garching, \\ Germany \\ E-mail: robert.schulzephysik.uni-wuerzburg.de
}

\begin{abstract}
Very-high-energy $\gamma$-ray observations of the active galaxy IC 310 with the MAGIC telescopes have revealed fast variability with doubling time scales of less than $4.8 \mathrm{~min}$. This implies that the emission region in IC 310 is smaller than $20 \%$ of the gravitational radius of the central supermassive black hole with a mass of $3 \times 10^{8} M_{\odot}$, which poses serious questions on the emission mechanism and classification of this enigmatic object. We report on the first quasi-simultaneous multifrequency VLBI observations of IC 310 conducted with the EVN. We find a blazar-like one-sided core-jet structure on parsec scales, constraining the inclination angle to be less than $\sim 20^{\circ}$ but very small angles are excluded to limit the de-projected length of the large-scale radio jet.
\end{abstract}

12th European VLBI Network Symposium and Users Meeting,

7-10 October 2014

Cagliari, Italy

\footnotetext{
* Speaker.
} 


\section{Introduction}

The active galaxy IC 310 is located at a distance of $z=0.0189$ [1] in the Perseus cluster and harbours a supermassive black hole with a mass of $3 \times 10^{8} M_{\odot}$ [2]. It was originally classified as a head-tail radio galaxy on kpc-scales [3]. However, Very Long Baseline Array (VLBA) snapshot observations at $8.3 \mathrm{GHz}$ revealed a blazar-like morphology on pc-scales with a single-sided jet extending from a dominating core [4]. It is part of the MOJAVE monitoring program ${ }^{1}$ observing with the VLBA at $15 \mathrm{GHz}$.

IC 310 is a source of high-energy emission up to the very-high $\gamma$-ray energies. It was detected above $30 \mathrm{GeV}$ by Fermi/LAT [5] and at TeV energies by the MAGIC telescopes [6]. In 2009 October and 2010 February MAGIC detected strong flux variability on time scales of one day, concluding that such a short-term variability requires a size of the very high energy emission zone of $\lesssim 80$ Schwarzschild-Radii [7].

A more recent MAGIC observation [2] in 2012 November revealed an even stronger highstate with multiple flares within a single night. The observation was part of a multiwavelength observation campaign including VLBI observations with the European VLBI Network (EVN). In the resulting first VLBI image of IC 310 at $5.0 \mathrm{GHz}$ counter-jet emission was not detected, yielding an upper limit of the angle of the jet to the line-of-sight of $20^{\circ}$. The $\mathrm{TeV}$ lightcurve measured by the MAGIC telescopes showed highly rapid flux variations with a doubling time scale down to $4.8 \mathrm{~min}$. Theoretical considerations to explain the short-term variability lead to a size of the emission zone of $20 \%$ of the gravitational radius of the supermassive black hole, which were interpreted in the framework of particle acceleration models similar to those used for pulsars.

\section{The EVN campaign}

The EVN observed IC 310 quasi-simultaneously at $1.7 \mathrm{GHz}, 5.0 \mathrm{GHz}, 8.4 \mathrm{GHz}$, and $22 \mathrm{GHz}$. The $5.0 \mathrm{GHz}$ image has been discussed previously [2]. Here, we present images at $1.7 \mathrm{GHz}$ and $8.4 \mathrm{GHz}$, while results from the $22 \mathrm{GHz}$ observation will be discussed elsewhere. The observation at $1.7 \mathrm{GHz}$ yield the first VLBI image of IC 310 at this frequency. The data were calibrated using standard routines in AIPS [8]. Gain factor corrections had to be determined manually for Jodrell Bank, Zelenchukskaya, and Badary. Hybrid imaging was performed using the software DIFMAP [9]. A list of the participating stations and the image parameters is given in Table 1.

The resulting new images are shown in Fig. 1 and reveal a one-sided jet similar to the $5.0 \mathrm{GHz}$ observation [2]. The sensitivity at $8.4 \mathrm{GHz}$ is improved over the snap-shot VLBA observation at $8.3 \mathrm{GHz}$ by a factor of $\sim 5$. The total flux density in the EVN images is consistent with a flat spectrum around $0.1 \mathrm{Jy}$. The brightness temperature of the prominent core at each frequency (including $5.0 \mathrm{GHz}$ ) was calculated to be of the order of $\sim 10^{10} \mathrm{~K}$ based on fitting elliptical Gaussian functions to the visibility data.

A comparison of the pc-scale (EVN) and kpc-scale morphology (NVSS, [10]) does not show any substantial variation of the position angle of the jet on de-projected scales from about $1 \mathrm{pc}$ out to about $500 \mathrm{kpc}$, which is consistent with previous studies [4].

\footnotetext{
${ }^{1}$ http://www.physics.purdue.edu/astro/MOJAVE/index.html
} 

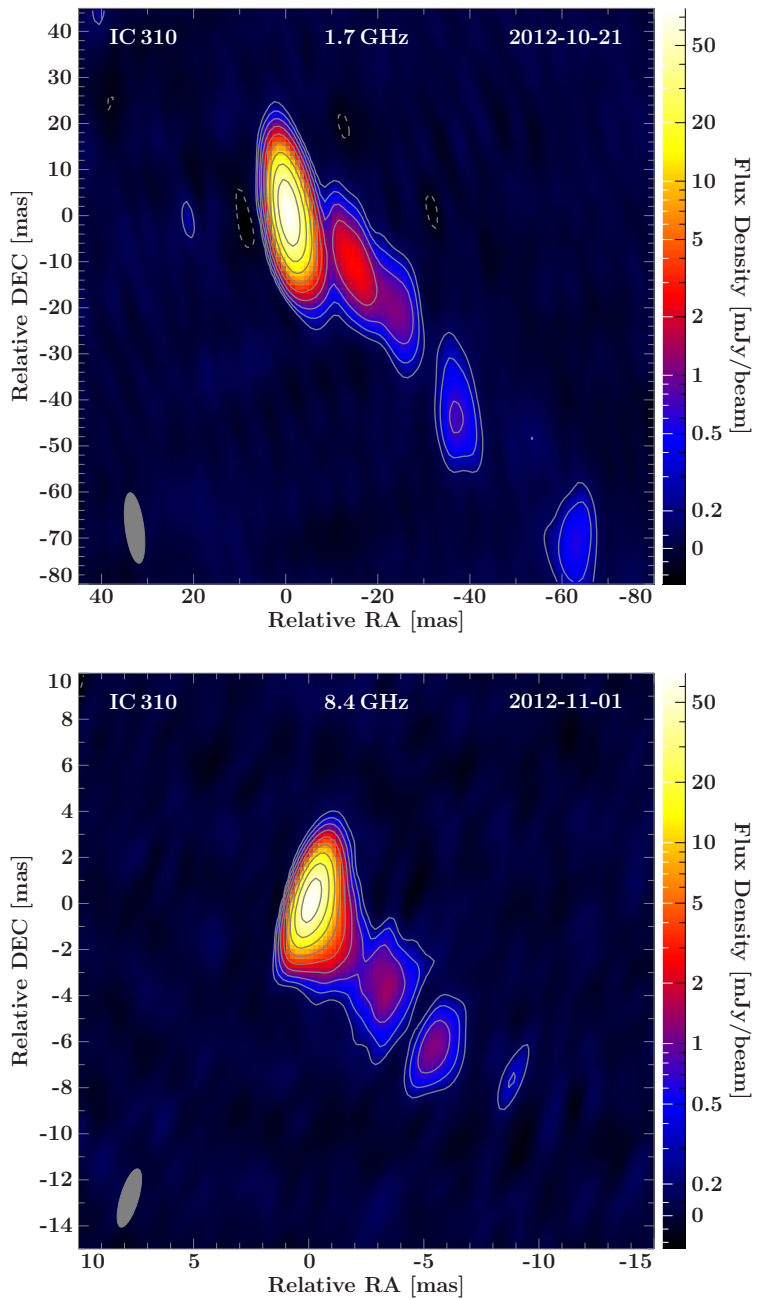

Figure 1: EVN images of IC 310 at $1.7 \mathrm{GHz}$ (top) and $8.4 \mathrm{GHz}$ (bottom). The contour lines start at five times the rms noise level $\sigma_{\text {rms }}$ (see Table 1) and increase logarithmically by factors of two. Negative contours are shown with dashed gray lines. The gray ellipse represents the synthesized beam. 1 mas correspond to a projected linear scale of $0.39 \mathrm{pc}$.

\section{Summary \& Outlook}

We present the first EVN images at $1.7 \mathrm{GHz}$ and $8.4 \mathrm{GHz}$ of the extremely variable $\mathrm{TeV}$ active galaxy IC 310 located in the Perseus cluster. The new VLBI data confirm the remarkably well collimated jet from pc-scales to kpc-scales and reveal a flat radio spectrum on parsec scales. The one-sided jet morphology extends from a high brightness temperature VLBI core. A detailed analysis of the full EVN campaign in particular in terms of the spectral index distribution and in combination with single-dish measurements will be featured in an upcoming publication (Schulz et al., in preparation).

\section{Acknowledgments}

R.S., C.M. and J.T. wish to thank RadioNet3 for support to attend this meeting. E.R. was partially 
Table 1: Overview of observation and image parameters

\begin{tabular}{|c|c|c|c|c|c|c|}
\hline$v$ & Date & Array $^{a}$ & $S_{\text {tot }}^{b}$ & $S_{\text {peak }}^{c}$ & $\sigma_{\mathrm{rms}}^{d}$ & Beam $^{e}$ \\
\hline$[\mathrm{GHz}]$ & [yyyy-mm-dd] & & [Jy] & [Jy/Beam] & [mJy/Beam] & {$[$ mas $\times$ mas, deg] } \\
\hline 1.7 & $2012-10-21$ & $\begin{array}{l}\text { EF WB JB ON MC NT TR } \\
\text { ZC BD UR SH }\end{array}$ & 0.10 & 0.078 & 0.033 & $15.4 \times 4.0,7.6$ \\
\hline 8.4 & 2012-11-01 & $\begin{array}{l}\text { EF WB ON MC YS SV ZC } \\
\text { BD UR }\end{array}$ & 0.10 & 0.070 & 0.037 & $2.6 \times 0.78,-16.7$ \\
\hline
\end{tabular}

a Antenna EF: Effelsberg, WB: Westerbork, JB: Jodrell Bank, ON: Onsala, MC: Medicina, NT: Noto, TR: Torun, ZC: Zelenchukskaya, BD: Badary, UR: Urumqi, SH: Sheshan, YS: Yebes, SV Svetloe; ${ }^{b}$ total flux density; ${ }^{c}$ peak flux density; ${ }^{d}$ noise level; ${ }^{e}$ beam parameters (major axis $\times$ minor axis, position angle)

supported by the Spanish MINECO project AYA2012-38491-C02-01 and by the Generalitat Valenciana project PROMETEOII/2014/057. We acknowledge support by the COST MP0905 action 'Black Holes in a Violent Universe'. The research leading to these results has received funding from the European Commission Seventh Framework Programme (FP/2007-2013) under grant agreement No. 283393 (RadioNet3). The European VLBI Network is a joint facility of European, Chinese, South African and other radio astronomy institutes funded by their national research councils. This research has made use of the Interactive Spectral Interpretation System (ISIS). This research has made use of a collection of ISIS scripts provided by the Dr. Karl Remeis observatory, Bamberg, Germany at http://www. sternwarte. uni-erlangen.de/isis/.

\section{References}

[1] M. Bernardi, M. V. Alonso, L. N da Costa, et al., Redshift-Distance Survey of Early-Type Galaxies. I. The ENEARc Cluster Sample, AJ 123 (2002) 2990

[2] J. Aleksić, S. Ansoldi, L. A. Antonelli, et al., Black hole lightning due to particle acceleration at subhorizon scales, Sci 346 (2014) 1080

[3] D. Sijbring, A. G. de Bruyn, Multifrequency radio continuum observations of head-tail galaxies in the Perseus cluster, A\&A 331 (1998) 901

[4] M. Kadler, D. Eisenacher, E. Ros, et al., The blazar-like radio structure of the TeV source IC 310, $A \& A 538(2012) \mathrm{L} 1$

[5] A. Neronov, D. Semikoz, I. Vovk, Very high-energy $\gamma$-ray emission from IC 310, A\&A $\mathbf{5 1 9}$ (2010) L6

[6] J. Aleksić, L. A. Antonelli, P. Antoranz, et al., Detection of Very High Energy $\gamma$-ray Emission from the Perseus Cluster Head-Tail Galaxy IC 310 by the MAGIC Telescopes, ApJ 723 (2010) L207

[7] J. Aleksić, L. A. Antonelli, P. Antoranz, et al., Rapid and multiband variability of the TeV bright active nucleus of the galaxy IC 310, A\&A $\mathbf{5 6 3}$ (2014) A91

[8] E. W. Greisen, AIPS, the VLA, and the VLBA, in Information Handling in Astronomy - Historical Vistas (2003)

[9] M. C. Shepherd, Difmap: an Interactive Program for Synthesis Imaging, in proceedings of Astronomical Data Analysis Software and Systems VI (1997)

[10] J. J. Condon, W. D. Cotton, E. W. Greisen, et al., The NRAO VLA Sky Survey, AJ 115 (1998) 1693 